\title{
Holomorphic Mappings of Domains in Operator Spaces
}

\author{
LAWRENCE A. HARRIS \\ Department of Mathematics, University of Kentucky, \\ Lexington, Kentucky 40506-0027, U. S. A.
}

\section{Introduction.}

Our object is to give an overview of some basic results about holomorphic mappings of circular domains in various spaces of operators. We begin by considering $\mathrm{C}^{*}$-algebras and pass to $\mathrm{J}^{*}$-algebras and other spaces when this seems natural.

Our first result is a simple extension of the maximum principle where the unitary operators play the role of the unit circle. We illustrate the power of this result by deducing some classical theorems of functional analysis in a straightforward way. Next we apply Cartan's uniqueness theorem to determine biholomorphic mappings and to show that linear mappings between certain operator domains are Jordan isomorphisms. This motivates the discussion of homogeneous domains.

Our first examples of homogeneous domains are the open unit balls of $\mathrm{J}^{*}$-algebras (which include all the classical domains.) Next we discuss some affinely homogeneous upper half-planes in spaces of operators called operator Siegel domains of genus 2. Although these are always holomorphically equivalent to a bounded domain, they are (as far as I know) not necessarily holomorphically equivalent to a ball. Our last examples are the domains of linear fractional transformations. These are symmetric affinely homogeneous domains which are never holomorphically equivalent to a bounded domain.

Finally we show how certain extensions of the Riemann removable singularity theorem allow us to determine the automorphisms of domains where an indefinite operator-valued form is positive. This includes the operator analogue of the exterior of the open unit disc.

\section{BASIC DEFINITIONS AND NOTATION.}

Let $X$ and $Y$ be complex normed linear spaces. Denote the open and closed unit balls of $X$ by $X_{0}$ and $X_{1}$, respectively. Thus,

$$
X_{0}=\{x \in X:\|x\|<1\}, \quad X_{1}=\{x \in X:\|x\| \leq 1\} .
$$

Let $\mathcal{D}$ be a domain in $X$. A function $h: \mathcal{D} \rightarrow Y$ is said to be holomorphic (in $\mathcal{D}$ ) if for each $x \in \mathcal{D}$ the Fréchet derivative of $h$ at $x$, denoted by $D h(x)$, exists and is a continuous complex-linear operator from $X$ to $Y$. If $\mathcal{D}^{\prime}$ is a domain in $Y$, a function $h: \mathcal{D} \rightarrow \mathcal{D}^{\prime}$ is said to be biholomorphic (and $\mathcal{D}$ and $\mathcal{D}^{\prime}$ are called holomorphically equivalent) if $h$ is a bijection and both $h: \mathcal{D} \rightarrow Y$ and $h^{-1}: \mathcal{D}^{\prime} \rightarrow X$ are holomorphic.

Let $\mathcal{L}(H, K)$ denote the Banach space of all bounded (complex-) linear operators from a Hilbert space $H$ to a Hilbert space $K$. The norm of $\mathcal{L}(H, K)$ is the operator norm and is given by $\|A\|=$ $\sup \left\{\|A x\|: x \in H_{1}\right\}$ for $A \in \mathcal{L}(H, K)$. We write $\mathcal{L}(H)$ for $\mathcal{L}(H, H)$. 
A $C^{*}$-algebra is a closed complex subspace $\mathfrak{A}$ of $\mathcal{L}(H)$ which contains the product of every two operators in $\mathfrak{A}$ and the adjoint of every operator in $\mathfrak{A}$. For example, the spaces $\mathcal{L}(H), \mathcal{L C}(H)$ and $\mathbb{C} I+\mathcal{L C}(H)$ are $\mathrm{C}^{*}$-algebras, where $\mathcal{L C}(H)$ denotes the set of compact linear operators on $H$. We say that $\mathfrak{A}$ is a $\mathrm{C}^{*}$-algebra with identity if, in addition, $\mathfrak{A}$ contains the identity operator $I$ on $H$. It is not difficult to show that if $S$ is a compact Hausdorff space, then the space $C(S)$ of all continuous complex-valued functions on $S$ is isometrically *-isomorphic to a commutative $\mathrm{C}^{*}$-algebra with identity. Thus the space $C(S)$ may be considered to be a $\mathrm{C}^{*}$-algebra with identity. From our point of view, $\mathrm{C}^{*}$-algebras are natural non-commutative analogues of the complex plane.

See [17] for the properties of holomorphic functions and the basic facts of functional analysis that we use. See [3] for a guide to the extensive literature on $\mathrm{C}^{*}$-algebras.

\section{The MAXIMUM PRINCIPLE AND APPLICATIONS.}

We begin with a useful theorem about holomorphic functions of operators which has some immediate applications to functional analysis. It has been presented in sharper and more general forms in [7] and [8].

Theorem 1. (Maximum principle.) Let $\mathfrak{A}$ be a $C^{*}$-algebra with identity, let $\mathcal{U}$ be the set of all unitary operators in $\mathfrak{A}$ and let $Y$ be a normed linear space. If $h: \mathfrak{A}_{1} \rightarrow Y$ is a continuous function that is holomorphic in $\mathfrak{A}_{0}$, then

$$
\|h(Z)\| \leq \sup _{U \in \mathcal{U}}\|h(U)\|
$$

for all $Z \in \mathfrak{A}_{1}$.

For example, let $\mathfrak{A}$ be the $\mathrm{C}^{*}$-algebra of all diagonal $n \times n$ matrices and note that $\mathfrak{A}$ may be identified with $\mathbb{C}^{n}$ with the max norm. Then $\mathfrak{A}_{0}$ is the open unit polydisc in $\mathbb{C}^{n}$ and $\mathcal{U}$ is its distinguished boundary. When a power series (about 0) in a single complex variable has radius of convergence $R>1$, the holomorphic functional calculus defines a holomorphic function as in Theorem 1. This leads to the following result.

Corollary 2. (von Neumann-Heins [5, p. 123].) If $f$ is a complex-valued function that is holomorphic in a neighborhood of the closed unit disc, then

$$
\|f(Z)\| \leq \sup _{|\lambda|=1}|f(\lambda)|
$$

for all $Z \in \mathcal{L}(H)$ with $\|Z\| \leq 1$.

Proof. If $U$ is a unitary operator in $\mathcal{L}(H)$, then $U$ is normal so $f(U)$ is normal. Hence, $\|f(U)\|=$ $|f(U)|_{\sigma}$, where $|\cdot|_{\sigma}$ denotes the spectral radius. By the spectral mapping theorem,

$$
|f(U)|_{\sigma}=\sup _{\lambda \in \sigma(U)}|f(\lambda)|,
$$

where $\sigma(\cdot)$ denotes the spectrum. Hence

$$
\|f(U)\| \leq \sup _{|\lambda|=1}|f(\lambda)|
$$

since $\sigma(U)$ is a subset of the unit circle. Corollary 2 now follows from Theorem 1 . 
Corollary 3. ( Russo-Dye [20].) The closed convex hull of $\mathcal{U}$ is $\mathfrak{A}_{1}$.

Proof. Let $\mathcal{K}$ be the closed convex hull of $\mathcal{U}$. Clearly $\mathcal{K} \subseteq \mathfrak{A}_{1}$. Suppose there exists a $Z \in \mathfrak{A}_{1}$ which is not in $\mathcal{K}$. By the Hahn-Banach separation theorem, there exists a continuous (complex-) linear functional $\ell$ on $\mathfrak{A}$ and a real constant $c$ such that $\operatorname{Re} \ell(U) \leq c$ for all $U \in \mathcal{U}$ and $\operatorname{Re} \ell(Z)>c$. But this contradicts the maximum principle for $h(W)=\exp (\ell(W))$.

Our proof of Theorem 1 (and other results below) uses linear fractional transformations of $\mathfrak{A}_{0}$ analogous to the linear fractional transformations of the open unit disc. (See [8, Theorem 2].)

Lemma 4. Let $\mathfrak{A}$ be a $C^{*}$-algebra and define

$$
T_{B}(Z)=\left(I-B B^{*}\right)^{-\frac{1}{2}}(Z+B)\left(I+B^{*} Z\right)^{-1}\left(I-B^{*} B\right)^{\frac{1}{2}}
$$

for each $B \in \mathfrak{A}_{0}$. Then $T_{B}$ is a biholomorphic mapping of $\mathfrak{A}_{0}$ onto itself and a homeomorphism of $\mathfrak{A}_{1}$ that takes 0 to $B$. If $I \in \mathfrak{A}$, then $T_{B}$ maps the set $\mathcal{U}$ onto itself.

Proof of Theorem 1. We may suppose that $Y=\mathbb{C}$. Indeed, by the Hahn-Banach theorem there exists a continuous linear functional $\ell$ on $Y$ with $\|\ell\|=1$ and $\ell(h(Z))=\|h(Z)\|$. Hence $(1)$ holds for $h$ if it holds when $h$ is replaced by the composition $\ell \circ h$.

Thus given $B \in \mathfrak{A}_{0}$, the complex-valued function $f(\lambda)=h\left(T_{B}(\lambda I)\right)$ is continuous in the closed unit disc and holomorphic in its interior. Hence by the classical maximum principle,

$$
|h(B)|=|f(0)| \leq \sup _{|\lambda|=1}|f(\lambda)| \leq \sup _{U \in \mathcal{U}}|h(U)|
$$

and (1) follows by continuity.

Special cases of Theorem 1 were given in [27, p. 284] and [25].

\section{Cartan's Uniqueness theorem and linear mappings.}

The next result is an extension to Banach spaces of a result of H. Cartan for $\mathbb{C}^{n}$. See [6], [23] and [10] for a discussion and proofs.

Theorem 5. (Cartan's uniqueness theorem.) Let $\mathcal{D}$ be a bounded domain in $X$ and let $h: \mathcal{D} \rightarrow \mathcal{D}$ be a holomorphic function. If there is a $p \in \mathcal{D}$ for which $h(p)=p$ and $D h(p)=I$, then $h=I$.

Every invertible linear isometry $L: X \rightarrow Y$ is a biholomorphic mapping of $X_{0}$ onto $Y_{0}$ taking 0 to 0 . The following shows that these are the only such mappings.

Corollary 6. If $h: X_{0} \rightarrow Y_{0}$ is a biholomorphic mapping with $h(0)=0$, then $h$ is the restriction to $X_{0}$ of an invertible linear isometry of $X$ onto $Y$.

Proof. Put $L=D h(0)$. By hypothesis and the chain rule, $L^{-1}=D h^{-1}(0)$. Thus applying the Cauchy estimates to $h$ and $h^{-1}$, we obtain $\|L\| \leq 1$ and $\left\|L^{-1}\right\| \leq 1$ so $L$ is an invertible isometry. Hence, Theorem 5 applies to the function $g=L^{-1} \circ h$ with $\mathcal{D}=X_{0}$ and $p=0$ to show that $g=I$, i.e., $h=L$.

Theorem 7. Let $\mathfrak{A}$ and $\mathfrak{B}$ be $C^{*}$-algebras. A function $h$ is a biholomorphic mapping of $\mathfrak{A}_{0}$ onto $\mathfrak{B}_{0}$ if and only if $h=T_{B} \circ L$, where $B \in \mathfrak{B}_{0}$ and $L: \mathfrak{A} \rightarrow \mathfrak{B}$ is an invertible linear isometry. 
Proof. Clearly $T_{B} \circ L$ is a biholomorphic mapping since it is the composition of two biholomorphic mappings. Conversely, if $h: \mathfrak{A}_{0} \rightarrow \mathfrak{B}_{0}$ is biholomorphic, let $B=h(0)$ and define $g=T_{B}^{-1} \circ h$. Then by Corollary 6, there is an invertible linear isometry $L: \mathfrak{A} \rightarrow \mathfrak{B}$ with $g=L$. Hence, $h=T_{B} \circ L$.

To discuss an application of Cartan's uniqueness theorem to linear mappings, let $\mathfrak{A}$ and $\mathfrak{B}$ be $\mathrm{C}^{*}$-algebras with identity, and set

$$
\mathfrak{A}_{\text {inv }}=\left\{Z \in \mathfrak{A}: Z^{-1} \text { exists }\right\}, \quad \mathfrak{A}_{\mathrm{rhp}}=\{Z \in \mathfrak{A}: \operatorname{Re} Z>0\},
$$

where $\operatorname{Re} Z=\left(Z+Z^{*}\right) / 2$. The set $\mathfrak{A}_{\text {rhp }}$ is thought of as the right half-plane of $\mathfrak{A}$. Clearly $\operatorname{Re} Z>0$ holds if and only if $\inf \operatorname{Re} W(Z)>0$, where $W(Z)$ denotes the numerical range of $Z$. Hence $\mathfrak{A}_{\text {rhp }} \subset$ $\mathfrak{A}_{\text {inv }}$ since the spectrum of an operator is contained in the closure of its numerical range. (Of course, this discussion applies to $\mathfrak{B}$ also.)

It is conjectured in [15] that if $L: \mathfrak{A} \rightarrow \mathfrak{B}$ is a linear bijection such that $L(I)=I$ and $L\left(\mathfrak{A}_{\text {inv }}\right)=$ $\mathfrak{B}_{\text {inv }}$, then $L\left(Z^{2}\right)=L(Z)^{2}$ for all $Z \in \mathfrak{A}$. (See also [1, p. 36-37].) The following result may be viewed as supporting this conjecture.

Proposition 8. If $L: \mathfrak{A} \rightarrow \mathfrak{B}$ is a linear bijection such that $L(I)=I$ and $L\left(\mathfrak{A}_{\mathrm{rhp}}\right)=\mathfrak{B}_{\mathrm{rhp}}$, then $L\left(Z^{2}\right)=L(Z)^{2}$ for all $Z \in \mathfrak{A}$.

Proof. We first show that $L$ is continuous. If $Z \in \mathfrak{A}_{0}$, then $I-\lambda Z$ is in $\mathfrak{A}_{\text {rhp }}$ for all $|\lambda| \leq 1$ so $I-\lambda L(Z)$ is in $\mathfrak{B}_{\text {rhp }}$ for all $|\lambda| \leq 1$. Hence, $|W(L(Z))|<1$, where $|W(\cdot)|$ denotes the numerical radius, so

$$
\|L(Z)\| \leq 2|W(L(Z))|<2 .
$$

Similarly, $L^{-1}$ is continuous. It follows that $L$ is a biholomorphic mapping of $\mathfrak{A}_{\text {rhp }}$ onto $\mathfrak{B}_{\text {rhp }}$.

Next note that since $\operatorname{Re} Z^{-1}=\left(Z^{-1}\right)^{*}(\operatorname{Re} Z) Z^{-1}$ for $Z \in \mathfrak{A}_{\text {inv }}$, the function $f(Z)=Z^{-1}$ is a biholomorphic mapping of $\mathfrak{A}_{\text {rhp }}$ (resp., $\mathfrak{B}_{\text {rhp }}$ ) onto itself with $D f(Z) W=-Z^{-1} W Z^{-1}$. Hence, the function

$$
h(Z)=L^{-1}\left(L\left(Z^{-1}\right)^{-1}\right)
$$

is a biholomorphic mapping of $\mathfrak{A}_{\mathrm{rhp}}$ onto itself with $h(I)=I$. By the chain rule, $D h(I)=I$. In order to apply Cartan's uniqueness theorem, it suffices to show that $\mathfrak{A}_{\text {rhp }}$ is holomorphically equivalent to a bounded domain. This is true since it is easy to verify that $T(Z)=(I-Z)(I+Z)^{-1}$ is a biholomorphic mapping of $\mathfrak{A}_{\text {rhp }}$ onto $\mathfrak{A}_{0}$.

Therefore, $h=I$, i.e., $L\left(Z^{-1}\right)=L(Z)^{-1}$ for all $Z \in \mathfrak{A}_{\text {rhp }}$. Taking the Fréchet derivative of each side of the last equality, we obtain

$$
L\left(-Z^{-1} W Z^{-1}\right)=-L(Z)^{-1} L(W) L(Z)^{-1}
$$

for all $Z \in \mathfrak{A}_{\text {rhp }}$ and $W \in \mathfrak{A}$. In particular, when $W=Z^{2}$, we obtain $L\left(Z^{2}\right)=L(Z)^{2}$ and this holds for all $Z \in \mathfrak{A}$ by the identity theorem.

Note that the above proof shows that if $h: \mathfrak{A}_{\mathrm{rhp}} \rightarrow \mathfrak{B}_{\mathrm{rhp}}$ is a biholomorphic mapping with $h(I)=I$, then $h\left(Z^{-1}\right)=h(Z)^{-1}$ for all $Z \in \mathfrak{A}_{\mathrm{rhp}}$. If Cartan's uniqueness theorem holds when $\mathcal{D}=\mathfrak{A}_{\mathrm{inv}}$ and $p=I$, then the above proof establishes the conjecture once we know that $L$ is continuous. 


\section{Generalizations of $\mathrm{C}^{*}$-Algebras.}

A careful examination of the linear fractional transformations $T_{B}$ shows that Lemma 4 holds for any closed complex subspace of $\mathcal{L}(H, K)$ such that $Z W^{*} Z \in \mathfrak{A}$ whenever $Z, W \in \mathfrak{A}$. Such spaces are called $J^{*}$-algebras. These were introduced in [8] and studied in [11] and [12], respectively. The above arguments show that Theorem 7 holds for $\mathrm{J}^{*}$-algebras and that Theorem 1 and Corollary 3 hold for $\mathrm{J}^{*}$-algebras containing the identity operator I. Proposition 8 holds for power algebras (defined in [6]) by the example preceding Proposition 12 below.

The main advantage of considering $\mathrm{J}^{*}$-algebras rather than $\mathrm{C}^{*}$-algebras is that the open unit balls of $\mathrm{J}^{*}$-algebras include all the classical Cartan domains [18], their infinite dimensional analogues and all finite and infinite products of these. To see this, recall that $\mathfrak{A}$ is called a Cartan factor of

Type I if $\mathfrak{A}=\mathcal{L}(H, K)$,

Type II if $\mathfrak{A}=\left\{A \in \mathcal{L}(H): A^{t}=A\right\}$,

Type III if $\mathfrak{A}=\left\{A \in \mathcal{L}(H): A^{t}=-A\right\}$,

Type IV if $A^{*} \in \mathfrak{A}$ and $A^{2} \in \mathbb{C} I$ whenever $A \in \mathfrak{A}$.

Here, $A^{t}=Q A^{*} Q$, where $Q(x)=\bar{x}$ is a conjugation on $H$. Any Hilbert space $H$ can be considered to be a Cartan factor of type I since $H$ is isometrically isomorphic to $\mathcal{L}(\mathbb{C}, H)$. It is shown in [8] that if $H$ is a Hilbert space with conjugation $x \rightarrow \bar{x}$, then there is a Cartan factor $\mathfrak{A}$ of type IV such that $\mathfrak{A}_{0}$ is linearly equivalent to the Lie ball

$$
\mathcal{D}=\left\{z \in H: 2\|z\|^{2}-|(z, \bar{z})|^{2}<1,|(z, \bar{z})|<1\right\} .
$$

Thus the Cartan domains of types I-IV are the open unit balls of the Cartan factors of the corresponding type.

It is easy to verify that the Cartan factors are $\mathrm{J}^{*}$-algebras. No Cartan factor is isomorphic to a product of two nonzero $\mathrm{J}^{*}$-algebras, except the two dimensional Cartan factor of type IV, and no two Cartan factors of different types are isomorphic, except for some cases in dimensions at most six. A J*-algebra is said to be of finite rank if it is a Hilbert space in an equivalent norm (as, for example, when it is finite dimensional.) Every $\mathrm{J}^{*}$-algebra of finite rank is isomorphic to finite product of Cartan factors of type I-IV.

The central idea of the theory is that questions about the holomorphic structure of the open unit ball of a $\mathrm{J}^{*}$-algebra reduce to corresponding questions about the algebraic structure of the $\mathrm{J}^{*}$-algebra, which can be attacked with the methods of functional analysis. For example, the open unit balls of two $\mathrm{J}^{*}$-algebras are holomorphically equivalent if and only if the $\mathrm{J}^{*}$-algebras are isomorphic. The open unit ball of a $\mathrm{J}^{*}$-algebra is not holomorphically equivalent to a product of balls of normed linear spaces if and only if the $\mathrm{J}^{*}$-algebra is not isomorphic to a product of two (nonzero) $\mathrm{J}^{*}$-algebras. The open unit ball of a $\mathrm{J}^{*}$-algebra has an invariant Hermitian metric if and only if the $\mathrm{J}^{*}$-algebra is of finite rank. The open unit ball ball of a $\mathrm{J}^{*}$-algebra is holomorphically equivalent to a Siegel domain of genus 2 (see [26]) if and only if the closed unit ball of the $\mathrm{J}^{*}$-algebra has an extreme point.

Call a domain $\mathcal{D}$ homogeneous if for any two points $p, q \in \mathcal{D}$ there exists a biholomorphic mapping $h: \mathcal{D} \rightarrow \mathcal{D}$ with $h(p)=q$. (If $h$ can always be chosen to be affine, we say that $\mathcal{D}$ is affinely homogeneous.) Call $\mathcal{D}$ symmetric if for each $p \in \mathcal{D}$ there exists a symmetry at $p$, i.e., a biholomorphic mapping $h: \mathcal{D} \rightarrow \mathcal{D}$ with $h(p)=p, h^{2}=I$ and $D h(p)=-I$. Note that any homogeneous ball is symmetric since for each $p \in \mathcal{D}$ there exists a biholomorphic mapping $T: \mathcal{D} \rightarrow \mathcal{D}$ with $T(0)=p$ and we may take $h(z)=T\left(-T^{-1}(z)\right)$ for $z \in \mathcal{D}$. For example, the open unit ball of any $\mathrm{J}^{*}$-algebra is a homogeneous and symmetric domain. However, there are two Banach spaces with dimension 16 and 27 , respectively, whose open unit balls are homogeneous domains that are not holomorphically equivalent to the open unit ball of any $\mathrm{J}^{*}$-algebra. To include these exceptional domains, Kaup 
$[21,22]$ defined an axiomatization of the triple product

$$
\{A, B, C\}=\frac{A B^{*} C+C B^{*} A}{2}
$$

on Banach spaces which he called JB*-triples and showed that every bounded symmetric domain is holomorphically equivalent to the open unit ball of a JB*-triple. See [19] for an exposition.

The results we have stated for $\mathrm{J}^{*}$-algebras continue to hold for $\mathrm{JB}^{*}$-triples. According to a Gelfand-Naimark type theorem of Friedman and Russo, every JB*-triple is isomorphic to the product of a $\mathrm{J}^{*}$-algebra and a subtriple of a space of continuous functions with values in the exceptional triple of dimension 27. See [2] and [28] for a guide to the extensive literature on JB*-triples.

\section{UPPER HALF-PLANES.}

Let $\mathfrak{A}$ be a $\mathrm{J}^{*}$-algebra and suppose $V$ is a partial isometry in $\mathfrak{A}$. (Equivalently, $V=V V^{*} V$ by [5, p. 63].) Then the generalized Cayley transform

$$
S_{V}(Z)=i\left(I+V V^{*}\right)^{-\frac{1}{2}}(Z+V)\left(I-V^{*} Z\right)^{-1}\left(I+V^{*} V\right)^{\frac{1}{2}}
$$

is a biholomorphic mapping of $\mathfrak{A}_{0}$ onto the convex homogeneous domain

$$
\mathcal{H}_{V}=\left\{Z \in \mathfrak{A}: 2 \operatorname{Im} V^{*} Z-Z^{*}\left(I-V V^{*}\right) Z+I-V^{*} V>0\right\},
$$

where $\operatorname{Im} Z=\left(Z-Z^{*}\right) /(2 i)$. For example, if $V=I$ then $\mathcal{H}_{V}=\{Z \in \mathfrak{A}: \operatorname{Im} Z>0\}$ and $\mathcal{H}_{V}$ is affinely homogeneous since

$$
A(Z)=\operatorname{Re} Z_{0}+\left(\operatorname{Im} Z_{0}\right)^{\frac{1}{2}} Z\left(\operatorname{Im} Z_{0}\right)^{\frac{1}{2}}
$$

is an affine biholomorphic mapping of $\mathcal{H}_{V}$ with $A(i I)=Z_{0}$ when $Z_{0} \in \mathcal{H}_{V}$. The next result shows that with even weaker conditions on $\mathfrak{A}$, if $\mathfrak{A}$ contains a "maximal" partial isometry $V$ then $\mathcal{H}_{V}$ is an affinely homogeneous Siegel domain of genus 2 .

Theorem 9. Let $\mathfrak{A}$ be a closed complex subspace of $\mathcal{L}(H, K)$. Suppose there exists a partial isometry $V$ in $\mathfrak{A}$ which is an extreme point of $\mathfrak{A}_{1}$ and such that $\mathfrak{A}$ contains $V Z^{*} W+W Z^{*} V$ whenever $Z$ and $W$ are in $\mathfrak{A}$. Then $\mathcal{H}_{V}$ is an affinely homogeneous convex domain which is holomorphically equivalent to a bounded domain. Moreover, there exists a $J^{*}$-algebra $\mathcal{X}$ in $\mathcal{L}(H)$ containing $I$ and a closed complex subspace $\mathcal{W}$ of $\mathcal{L}\left(K_{1}, H\right) \times \mathcal{L}\left(H, K_{2}\right)$, which contains $\left(X W_{1}, W_{2} X\right)$ whenever $X$ is in $\mathcal{X}$ and $\left(W_{1}, W_{2}\right)$ is in $\mathcal{W}$, such that $\mathcal{H}_{V}$ is linearly equivalent to

$$
\mathcal{D}=\{(X, W) \in \mathcal{X} \times \mathcal{W}: 2 \operatorname{Im} X-F(W, W)>0\},
$$

where $F: \mathcal{W} \times \mathcal{W} \rightarrow \mathcal{X}$ is the operator-valued Hermitian map given by

$$
F(W, W)=W_{1} W_{1}^{*}+W_{2}^{*} W_{2}, \quad W=\left(W_{1}, W_{2}\right) .
$$

The extreme points of $\mathfrak{A}_{1}$ are the partial isometries $V \in \mathfrak{A}$ satisfying $\left(I-V V^{*}\right) Z\left(I-V^{*} V\right)=0$ for all $Z \in \mathfrak{A}$. There is at least one extreme point when $\mathfrak{A}$ is closed in the weak operator topology as, for example, when $\mathfrak{A}$ is a Cartan factor or a $J^{*}$-algebra of finite rank.

The domain $\mathcal{D}$ of the above theorem is a special kind of Siegel domain of genus 2 called an operator Siegel domain. See [9] for a proof of the above theorem and further details. A discussion of the generalized Cayley transform and $\mathcal{H}_{V}$ when $V$ is not necessarily a partial isometry can be found in [13, Theorem 11] (where the sign in the definition of $B$ should be omitted). 


\section{Domains of Linear FraCtional transformations.}

We begin with some facts about linear fractional transformations given in [13]. If $M \in \mathcal{L}(K \times H)$, let $M=\left[\begin{array}{cc}A & B \\ C & D\end{array}\right]$ be the corresponding partition and define the linear fractional transformation with coefficient matrix $M$ to be

$$
T(Z)=(A Z+B)(C Z+D)^{-1} .
$$

Clearly, if $Z \in \mathcal{L}(H, K)$ and $T(Z)$ exists, then $T(Z) \in \mathcal{L}(H, K)$. To avoid degeneracy, we always assume that $M$ is invertible. We write $T_{M}$ for $T$ when we wish to indicate the dependence on $M$. It can be shown that $T_{M}^{-1}=T_{M^{-1}}$ and, in particular, that both these transformations have the same domain of definition.

Let $\mathfrak{A}$ and $\mathfrak{B}$ be any closed complex subspaces of $\mathcal{L}(H, K)$ and suppose $T$ is defined at some point $Z_{0} \in \mathfrak{A}$. Put $W_{0}=T\left(Z_{0}\right), X_{0}=\left(C Z_{0}+D\right)^{-1} C$,

$$
\begin{aligned}
& \mathcal{E}_{1}=\operatorname{Comp}_{Z_{0}}\left(\left\{Z \in \mathfrak{A}:(C Z+D)^{-1} \text { exists }\right\}\right), \\
& \mathcal{E}_{2}=\operatorname{Comp}_{W_{0}}\left(\left\{W \in \mathfrak{B}:(A-W C)^{-1} \text { exists }\right\}\right),
\end{aligned}
$$

where $\operatorname{Comp}_{p}(S)$ denotes the topological component of $S$ containing $p$.

Theorem 10. The following are equivalent:

a) $T$ maps a domain $\mathcal{D}$ in $\mathfrak{A}$ into $\mathfrak{B}$ and the closure of $T(\mathcal{D})$ contains some ball in $\mathfrak{B}$,

b) $T$ is a biholomorphic mapping of $\mathcal{E}_{1}$ onto $\mathcal{E}_{2}$,

c) $\mathfrak{B}=\left(A-W_{0} C\right) \mathfrak{A}\left(C Z_{0}+D\right)^{-1}, W_{0} \in \mathfrak{B}$ and $Z X_{0} Z \in \mathfrak{A}$ whenever $Z \in \mathfrak{A}$.

We define a domain $\mathcal{D}$ to be the domain of a linear fractional transformation in $\mathfrak{A}$ if $\mathcal{D}=\mathcal{E}_{1}$ for some $T$ satisfying the hypotheses of Theorem 10 and this is equivalent to the condition that $Z X_{0} Z \in \mathfrak{A}$ whenever $Z \in \mathfrak{A}$ since the other conditions of part (c) are satisfied, for example, when $T(Z)=\left(Z-Z_{0}\right)\left[I+X_{0}\left(Z-Z_{0}\right)\right]^{-1}$. (See [14].) Clearly, $\mathcal{E}_{2}$ is also the domain of a linear fractional transformation (e.g., $T^{-1}$ ) in $\mathfrak{B}$. If $\mathfrak{A}$ is finite dimensional, $\mathcal{D}$ is the complement of the algebraic variety in $\mathbb{C}^{n}$ defined by $\operatorname{det}(C Z+D)=0$.

Theorem 11. The domain of a linear fractional transformation is a symmetric, affinely homogeneous domain.

Proof. Given $Y \in \mathcal{D}$, define

$$
U_{Y}(Z)=Y-(Z-Y)(C Z+D)^{-1}(C Y+D)
$$

for $Z \in \mathcal{D}$. It is not difficult to deduce from Theorem 10 and the identity

$$
C U_{Y}(Z)+D=(C Y+D)(C Z+D)^{-1}(C Y+D)
$$

that $U_{Y}$ is a symmetry at $Y$. Hence $\mathcal{D}$ is symmetric. Given $Z_{0}, W_{0} \in \mathcal{D}$ with $\left\|X_{0}\left(W_{0}-Z_{0}\right)\right\|<1$, define

$$
\phi(Z)=W_{0}+\left[I+\left(W_{0}-Z_{0}\right) X_{0}\right]^{\frac{1}{2}}\left(Z-Z_{0}\right)\left[I+X_{0}\left(W_{0}-Z_{0}\right)\right]^{\frac{1}{2}},
$$


where the square roots are defined by the binomial series. Similar methods show that the affine mapping $\phi$ is a biholomorphic mapping of $\mathcal{D}$ with $\phi\left(Z_{0}\right)=W_{0}$. If $Z_{0}, W_{0} \in \mathcal{D}$ are arbitrary, there is a sequence $Z_{1}, \ldots, Z_{n}$ in $\mathcal{D}$ with

$$
\left\|\left(C Z_{k-1}+D\right)^{-1} C\left(Z_{k}-Z_{k-1}\right)\right\|<1, \quad k=1, \ldots, n,
$$

and $Z_{n}=W_{0}$. Hence if $\phi_{k}$ is the mapping constructed above with $\phi_{k}\left(Z_{k-1}\right)=Z_{k}$, the composition $h=\phi_{n} \circ \cdots \circ \phi_{1}$ is a biholomorphic mapping of $\mathcal{D}$ with $h\left(Z_{0}\right)=W_{0}$. Therefore, $D$ is affinely homogeneous.

For example, let $\mathfrak{A}=\mathcal{L}(H, K), C \in \mathcal{L}(K, H)$ and $D \in \mathcal{L}(H)$, and consider $\mathcal{D}=\{Z \in \mathfrak{A}:(C Z+$ $D)^{-1}$ exists $\}$. Then $\mathcal{D}$ is the domain of a linear fractional transformation when $\mathcal{D}$ is non-empty and the range of $C$ is closed (since then $\mathcal{D}$ is connected). In particular, $\mathcal{D}=\mathfrak{A}$ and $U_{Y}(Z)=2 Y-Z$, when $C=0$ and $D=I$. Any Hilbert space $H$ can be identified with $\mathfrak{A}=\mathcal{L}(\mathbb{C}, H)$ and, under this identification, $\mathcal{D}=\{z \in H:(z, c) \neq-d\}$, where $c \in H$ and $d \in \mathbb{C}$. Hence $\mathcal{D}$ is the domain of a linear fractional transformation if $c \neq 0$.

For another example, let $\mathfrak{A}$ be a closed complex subspace of $\mathcal{L}(H)$ containing $I$, take $Z_{0}=I$ and put $\mathcal{D}=\operatorname{Comp}_{I}\left\{Z \in \mathfrak{A}: Z^{-1}\right.$ exists $\}$. Then $\mathcal{D}$ is the domain of a linear fractional transformation if and only if $Z^{2} \in \mathfrak{A}$ whenever $Z \in \mathfrak{A}$, i.e., $\mathfrak{A}$ is a power algebra. In this case, $U_{Y}(Z)=Y Z^{-1} Y$. Under the identification of the Hilbert space $\mathbb{C}^{n}$ with the associated Cartan factor of type IV, we have $\mathcal{D}=\left\{z \in \mathbb{C}^{n}: z \cdot z \neq 0\right\}$ and

$$
U_{y}(z)=\frac{2(z \cdot y) y-(y \cdot y) z}{z \cdot z} .
$$

Domains of linear fractional transformations are very much unlike the previous homogeneous domains we have considered since they are not holomorphically equivalent to any bounded domains by the next result. (See [14, Theorem 2].)

Proposition 12. Every bounded holomorphic function on the domain of a linear fractional transformation is constant.

Let $\mathcal{D}$ be the domain of a linear fractional transformation in $\mathfrak{A}$. An important question is whether every bounded holomorphic function $h: \mathcal{D} \cap \mathfrak{A}_{0} \rightarrow \mathbb{C}$ extends to $\mathfrak{A}_{0}$ when $\mathcal{D}$ intersects $\mathfrak{A}_{0}$. This is true when $\mathfrak{A}$ is finite dimensional by the Riemann removable singularities theorem. This is also true when $C$ is a trace class operator by [14, Theorem 3] and when $C=I, D=0$ and $\mathfrak{A}$ is $\mathrm{W}^{*}$-closed by $[14$, Proposition 8]. We apply these facts in the next section. (Note that Proposition 12 is a consequence of the general case, if true.)

\section{Determination of automorphisms.}

Let $\mathfrak{A}=\mathcal{L}(H, K)$, where $H$ and $K$ are Hilbert spaces with different dimensions. As we learned, Theorem 7 holds for this space. It can be shown that any invertible linear isometry $L: \mathfrak{A} \rightarrow \mathfrak{A}$ is of the form $L(Z)=U Z V$, where $U \in \mathcal{L}(K)$ and $V \in \mathcal{L}(H)$ are unitary operators. (The assumption of different dimensions excludes the map $L(Z)=Z^{t}$.) Thus the following is obtained.

Theorem 13. (Greenfield-Wallach-Franzoni [4].) A function $h: \mathfrak{A}_{0} \rightarrow \mathfrak{A}_{0}$ is a biholomorphic mapping of $\mathfrak{A}_{0}$ if and only if $h=T_{M}$, where $M$ is a J-unitary operator in $\mathcal{L}(K \times H)$, i.e., $M$ is invertible and $M^{*} J M=J$, where $J=\left[\begin{array}{cc}I & 0 \\ 0 & -I\end{array}\right]$. 
Theorem 14. Suppose $\mathfrak{A}=\mathcal{L}(H, K \times H)$, where $H$ and $K$ are Hilbert spaces with $K \neq\{0\}$ and $H$ is finite dimensional. Let

$$
\mathcal{D}=\left\{\left[\begin{array}{l}
Z_{1} \\
Z_{2}
\end{array}\right] \in \mathfrak{A}: I+Z_{1}^{*} Z_{1}<Z_{2}^{*} Z_{2}\right\} .
$$

Then a function $h: \mathcal{D} \rightarrow \mathcal{D}$ is a biholomorphic mapping if and only if $h(Z)=M Z U$, where $M$ is a $J$-unitary operator in $\mathcal{L}(K \times H)$ and $U$ is a unitary operator in $\mathcal{L}(H)$.

Proof. Let $T$ be the linear fractional transformation on $\mathfrak{A}$ defined by

$$
T\left[\begin{array}{l}
Z_{1} \\
Z_{2}
\end{array}\right]=\left[\begin{array}{c}
Z_{1} Z_{2}^{-1} \\
Z_{2}^{-1}
\end{array}\right]
$$

and let $\mathcal{E}$ be the domain of $T$. It is easy to check that $T$ is a biholomorphic mapping of $\mathcal{D}$ onto $\mathfrak{A}_{0} \cap \mathcal{E}$ with $T^{-1}=T$. Given a biholomorphic mapping $h$ of $\mathcal{D}$, define $f=T \circ h \circ T^{-1}$ and note that $f$ is a biholomorphic mapping of $\mathfrak{A}_{0} \cap \mathcal{E}$. Since $H$ is finite dimensional, the coefficient $C=\left[\begin{array}{ll}0 & I\end{array}\right]$ of $T$ is a trace class operator and thus $f$ extends to a biholomorphic mapping of $\mathfrak{A}_{0}$. Hence by the previous theorem, $f(Z)=(A Z+B)(C Z+D)^{-1}$, where $\tilde{M}=\left[\begin{array}{cc}A & B \\ C & D\end{array}\right]$ is a J-unitary operator in $\mathcal{L}((K \times H) \times H)$. Also, $f^{-1}(Z)=\left(A^{*} Z-C^{*}\right)\left(-B^{*} Z+D^{*}\right)^{-1}$ since $\tilde{M}^{-1}=J \tilde{M}^{*} J$.

By the mapping properties of $f$, if $Z \in \mathfrak{A}_{0}$, the second component $(A Z+B)_{2}$ is invertible or singular according as $Z_{2}$ is invertible or singular, and a similar result holds for $f^{-1}$. Since such mappings are linear in $Z_{2}$ (see [14, p. 462] and compare [15, Corollary 4.2]), we have that

$$
A=\left[\begin{array}{cc}
A_{1} & 0 \\
0 & A_{4}
\end{array}\right], B=\left[\begin{array}{c}
B_{1} \\
0
\end{array}\right], C=\left[\begin{array}{ll}
C_{1} & 0
\end{array}\right] .
$$

Since $h=T^{-1} \circ f \circ T$, it follows that $h(Z)=M Z U$, where $M=\left[\begin{array}{cc}A_{1} & B_{1} \\ C_{1} & D\end{array}\right]$ and $U=A_{4}{ }^{-1}$. Moreover, $M$ is J-unitary and $U$ is unitary since $\tilde{M}$ is J-unitary.

The converse assertion is easily verified.

An argument with a similar approach establishes the following result.

Theorem 15. Suppose $\mathfrak{A}$ is a $W^{*}$-closed subspace of $\mathcal{L}(H)$ which contains the squares of each of its elements and the identity operator I on $H$. Let $\mathfrak{A}_{\mathrm{inv}}^{e}$ denote the identity component of the set of all invertible operators in $\mathfrak{A}$ and put

$$
\mathcal{D}=\left\{Z \in \mathfrak{A}_{\mathrm{inv}}^{e}: I<Z^{*} Z\right\} .
$$

Then $h$ is a biholomorphic mapping of $\mathcal{D}$ onto itself if and only if $h=L$, where $L$ is a linear isometry of $\mathfrak{A}$ onto itself with $L(I) \in \mathfrak{A}_{\mathrm{inv}}^{e}$.

See [14] for further details and results. 
Throughout, most of the domains we have considered are circular domains in the sense of [13]. Specifically, if $\mathfrak{A}$ is a closed complex subspace of of $\mathcal{L}(H, K)$, a circular domain in $\mathfrak{A}$ is a set of the form

$$
\mathcal{D}=\left\{Z \in \mathfrak{A}: Z^{*} E Z+2 \operatorname{Re} F^{*} Z+G<0\right\},
$$

where $E \in \mathcal{L}(K), F \in \mathcal{L}(H, K), G \in \mathcal{L}(H)$ and both $E$ and $G$ are self adjoint. For example, $\mathfrak{A}_{0}$ is a circular domain since $\mathfrak{A}_{0}=\left\{Z \in \mathfrak{A}: Z^{*} Z-I<0\right\}$. The circular domains of the complex plane are any open disc, the exterior of any open disc, any open half-plane, any punctured plane, the entire plane and the empty set. (This terminology is taken from [24, p. 57] and [16, p. 464]. A different definition, which is also referred to as "circled," is given in [19, p. 113] and [2, p. 104].)

One of our main objectives has been to determine the automorphisms of circular domains and to discover circular domains that are homogeneous. Linear fractional transformations have served as a basic tool.

\section{References}

[1] B. Aupetit and H. du T. Mouton, Spectrum preserving linear mappings in Banach Algebras, Studia Math. 109(1994), 91-100.

[2] S. Dineen, The Schwarz Lemma, Oxford Math. Monographs, Oxford, 1989.

[3] J. Dixmier, $C^{*}$-algebras, North Holland, Amsterdam, 1977.

[4] T. Franzoni, The group of holomorphic automorphisms in certain $J^{*}$-algebras, Ann. Mat. Pura Appl. 127(1981), 51-66.

[5] P. R. Halmos, A Hilbert Space Problem Book, Van Nostrand, Princeton, 1967.

[6] L. A. Harris, Schwarz's lemma in normed linear spaces, Proc. Nat. Acad. Sci. U.S.A. 62(1969), $1014-1017$.

[7] Banach algebras with involution and Möbius transformations, J. Functional Anal. 11(1972), 1-16.

[8] B _ Bounded symmetric homogeneous domains in infinite dimensional spaces, in Infinite Dimensional Holomorphy, Lecture Notes in Math., Vol. 364, 13-40, Springer-Verlag, Berlin, 1974.

[9] - Operator Siegel domains, Proc. Royal Soc. Edinburgh Sect. 79A (1977), 135-156.

[10] , Schwarz-Pick systems of pseudometrics for domains in normed linear spaces, in Advances in Holomorphy, J. A. Barroso, ed., North-Holland, Amsterdam, 1979, pp. 345-406.

[11] _ Analytic invariants and the Schwarz-Pick inequality, Israel J. Math 34(1979), 177197.

$[12] \longrightarrow$ A generalization of $C^{*}$-algebras, Proc. London Math. Soc. 42(1981), 331-361.

[13] Linear fractional transformations of circular domains in operator spaces, Indiana Univ. Math. J. 41 (1992), 125-147. 
[14] Unbounded symmetric homogeneous domains in spaces of operators, Ann. Scuola Norm. Sup. Pisa 22(1995), 449-467.

[15]_ and R. V. Kadison, Affine mappings of invertible operators, Proc. Amer. Math. Soc. 124(1996), 2415-2422.

[16] P. Henrici, Applied and Computational Complex Analysis, Vol I, Wiley-Interscience, New York, 1974.

[17] E. Hille and R. S. Phillips, Functional Analysis and Semi-Groups, Amer. Math. Soc. Colloq. Publ., Vol. 31, AMS, Providence, 1957.

[18] L. K. Hua, Harmonic Analysis of Functions of Several Complex Variables in the Classical Domains, AMS, Providence, 1963.

[19] J.M. Isidro and L.L. Stachó, Holomorphic Automorphism Groups in Banach Spaces: An Elementary Introduction, Mathematics Studies Vol. 105, North-Holland, Amsterdam, 1985.

[20] R. V. Kadison and G. K. Pedersen, Means and convex combinations of unitary operators, Math. Scand. 57(1985), 249-266.

[21] W. Kaup, Algebraic characterization of symmetric complex manifolds, Math. Ann. 228(1977), $39-64$.

[22] _ A Riemann mapping theorem for bounded symmetric domains in complex Banach spaces, Math. Z. 183(1983), 503-529.

[23] _ and H. Upmeier, Banach spaces with biholomorphically equivalent unit balls are isomorphic, Proc. Amer. Math. Soc. 58(1976), 129-133.

[24] M. Marden, Geometry of Polynomials, Math. Surveys 3, Amer. Math. Soc., Providence, 1966.

[25] E. Nelson, The distinguished boundary of the unit operator ball, Proc. Amer. Math. Soc. 12(1961), 994-995.

[26] I.I. Pyatetskii-Shapiro, The Geometry of the Classical Domains and the Theory of Automorphic Functions, Fizmatgiz, Moscow 1961; French transl. Donod, Paris 1966; English transl. Gordon and Breach, New York 1969.

[27] R. M. Redheffer, On a certain linear fractional transformation, J. Math. and Physics 39(1960), 269-286.

[28] B. Russo, Structure of JB*-triples, in Jordan Algebras, Oberwolfach, 1992, ed. by W. Kaup et. al., 208-280, de Gruyter, Berlin, 1994. 